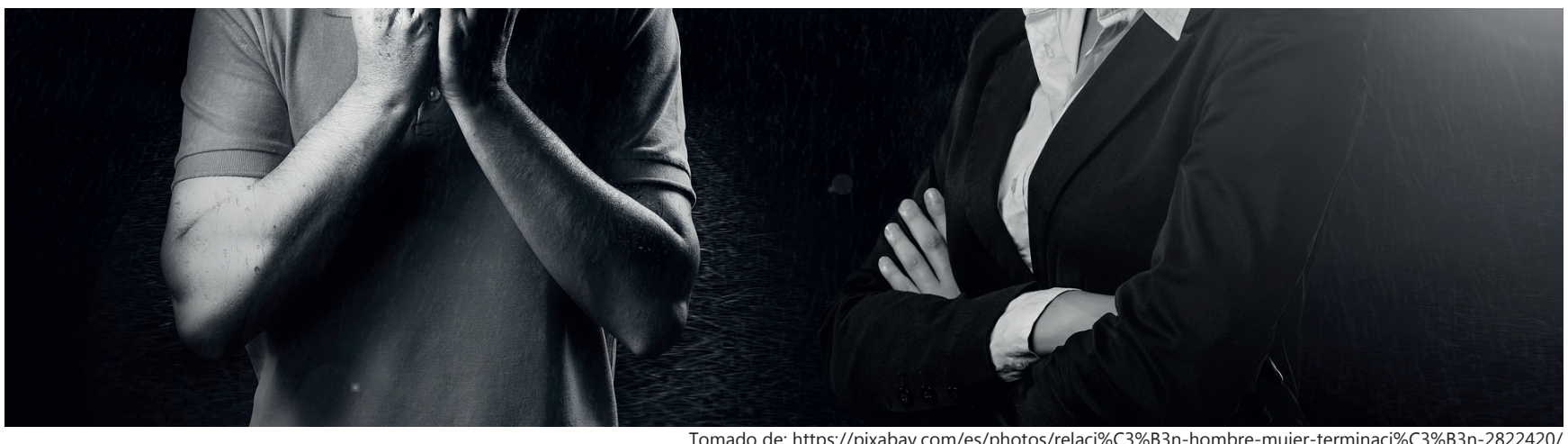

\title{
Libre desarrollo de la personalidad con perspectiva de derecho emergente
}

\section{Free personality development with an emerging law perspective}

\author{
Cielo Aracely Villarreal-Garza*, Antonio de Jesús Ramírez-Aguilar
}

\begin{abstract}
Resumen
A partir del concepto que la Suprema Corte de Justicia de la Nación ha establecido en su tesis "Derecho al libre desarrollo de la personalidad. Brinda protección a un área residual de libertad que no se encuentra cubierta por las otras libertades públicas", publicada en el Diario Oficial de la Federación en febrero de 2019, vale la pena reflexionar sobre sus alcances y preguntarnos si el libre desarrollo de la personalidad es un derecho emergente. Para ello, precisamos qué se entiende por derechos humanos emergentes, los motivos y justificación de su surgimiento, sus alcances e implicaciones, expuestos por el Instituto de Derechos Humanos de Cataluña, impulsor de este movimiento. Enseguida, revisamos la postura de la Suprema Corte de Justicia de la Nación acerca del derecho humano del libre desarrollo de la personalidad, que a través de su jurisprudencia gradualmente ha ido matizando. Hacemos énfasis en la sentencia que consideramos que ha reunido los distintos elementos de los que se compone este derecho, para analizarlos y dar respuesta al cuestionamiento inicial.
\end{abstract}

Palabras clave: Derechos humanos emergentes, libre desarrollo de la personalidad, jurisprudencia, Suprema Corte de Justicia de la Nación.

\begin{abstract}
Based on the concept that the Supreme Court of Justice of the Nation has established in its thesis "Right to the free development of personality. It provides protection to a residual area of freedom that is not covered by other public liberties", published in the Official Gazette of the Federation in February 2019, it is worth reflecting on its scope and asking ourselves if the free development of personality is an emerging right. To do this, we need to understand what is understood by emerging human rights, the reasons and justification for its emergence, its scope and implications, set out by the Institute of Human Rights of Catalonia, promoter of this movement. Next, we review the position of the Supreme Court of Justice of the Nation regarding the human right of the free development of the personality, which through its jurisprudence has gradually been nuanced. We emphasize the judgment that we consider has brought together the different elements of which this right is composed, to analyze them and respond to the initial questioning.
\end{abstract}

Keywords: Emerging human rights, free development of personality, jurisprudence, Supreme Court of Justice of the Nation. 


\section{INTRODUCCIÓN}

Discusiones académicas contemporáneas aluden a qué son los derechos humanos, su contenido, alcance y aplicación, resultando de ello el debate histórico sobre lo que es la justicia. Unos desde el punto de vista de que el derecho deriva de entes metafísicos o divinos y que lo que es acorde con lo natural es justo. Otros desde la perspectiva opuesta, consistente en que el derecho no deriva de la naturaleza (iusnaturalistas) sino del legislador, y que aun cuando una norma no sea acorde a la naturaleza, sigue siendo derecho (iuspositivismo).

En la historia, grandes acontecimientos han generado concepciones del derecho y de los derechos, como han sido los derivados de la Revolución Francesa y de la Segunda Guerra Mundial. Estos sucesos muestran que algunos derechos estaban sumergidos e irrumpieron, siendo materia de debates tanto de la academia como de la sociedad y los tribunales.

En el caso de los actuales derechos humanos emergentes, diversos doctrinarios especializados han tratado de definirlos, pero el Instituto de Derechos Humanos de Cataluña, en la Declaración Universal de Derechos Humanos Emergentes (2007) lo expone de forma clara y sencilla: "Los derechos humanos emergentes son reivindicaciones legítimas de la sociedad civil dirigidas a la formulación de nuevos o renovados derechos humanos". Esto implica que hay derechos que se han añejado o acartonado con el paso del tiempo y requieren ser actualizados en consonancia con la evolución de la sociedad y sus necesidades, mientras que otros ni siquiera fueron contemplados en el pasado y que el surgimiento de nuevos requerimientos de la sociedad obliga a incluirlos.

Durante la segunda versión del evento Fórum Universal de las Culturas, desarrollado en 2007 en Monterrey, México, fue aprobado por los representantes de la sociedad civil el documento denominado Declaración Universal de Derechos Humanos Emergentes (DUDHE). En éste se plasmó lo que se estimó una actualización de la Declaración Universal de Derechos
Humanos de 1948, complementada por la perspectiva de la ciudadanía participativa y considerando la necesidad del reconocimiento de derechos que trae consigo la realidad de los tiempos que vivimos; es decir, está orientado en el diseño de derechos colectivos y de los pueblos en las sociedades contemporáneas, tomando en cuenta que los derechos humanos son la base de toda sociedad. Dicho documento se construye desde las diversas experiencias y luchas de la sociedad civil global, recogiendo las reivindicaciones más perfiladas de sus movimientos sociales, trayendo consigo la plena e invaluable participación ciudadana.

La Declaración tiene por objeto fortalecer la interdependencia e integridad de los derechos de hombres y mujeres:

No pretende reemplazar ningún instrumento existente, al contrario, los completa y refuerza. Se trata de una Declaración que emana de la sociedad mundial global y debe de ser considerada como parte de un proceso normativo consuetudinario, pero también debe de ser considerada para los individuos y los Estados como un nuevo imperativo ético del siglo XXI (Declaración Universal de Derechos Humanos Emergentes, 2007).

Esta declaración está integrada por un breviario que indica su surgimiento y objetivo, los valores y principios que toma como parámetro y un total de 47 (cuarenta y siete) derechos, con la democracia como eje central.

\section{Libre desarrollo de la personalidad}

El derecho al libre desarrollo de la personalidad es la facultad que cada individuo tiene para elegir de manera autónoma su forma de vivir. Este derecho garantiza a los sujetos plena independencia para escoger, por ejemplo, su profesión, estado civil, pasa tiempos, apariencia física, estudios o actividad laboral, y solo está limitado por el respeto a los demás y el interés general. Mediante esta prerrogativa el Estado reconoce la facultad de toda persona de elegir ser y actuar de la manera que mejor le convenga para cumplir 
con sus preferencias, metas y expectativas particulares de vida. (Hernández, 2018).

Es decir, consiste en la capacidad de tomar decisiones de forma autónoma, lo que le sirve para definir su propio plan de vida (Carbonell, 2008, p. 211).

Este derecho está consagrado de manera específica en la Declaración Universal de los Derechos Humanos (DUDH), documento acogido por la Asamblea General de las Naciones Unidas en diciembre de 1948, en París. Esta declaración, fruto del terrible impacto que dejó la Segunda Guerra Mundial, marca un ideal común de paz y bienestar para todas las naciones. En sus artículos 10, 22 y 26 expresa:

Artículo $1^{\circ}$

Todos los seres humanos nacen libres e iguales en dignidad y derechos y, dotados como están de razón y conciencia, deben comportarse fraternalmente los unos con los otros.

Artículo 22

Toda persona, como miembro de la sociedad, tiene derecho a la seguridad social, y a obtener, mediante el esfuerzo nacional y la cooperación internacional, habida cuenta de la organización y los recursos de cada Estado, la satisfacción de los derechos económicos, sociales y culturales, indispensables a su dignidad y al libre desarrollo de su personalidad.

Artículo 26

1. Toda persona tiene derecho a la educación. La educación debe ser gratuita, al menos en lo concerniente a la instrucción elemental y fundamental. La instrucción elemental será obligatoria. La instrucción técnica y profesional habrá de ser generalizada; el acceso a los estudios superiores será igual para todos, en función de los méritos respectivos.

2. La educación tendrá por objeto el pleno desarrollo de la personalidad humana y el fortalecimiento del respeto a los derechos humanos y a las libertades fundamentales; favorecerá la comprensión, la tolerancia y la amistad entre todas las naciones y todos los grupos étnicos o religiosos, y promoverá el desarrollo de las actividades de las Naciones Unidas para el mantenimiento de la paz.

3. Los padres tendrán derecho preferente a escoger el tipo de educación que habrá de darse a sus hijos (Declaración Universal de Derechos Humanos de 1948).

La Revolución Francesa, en 1789, fue un parteaguas en la historia de la humanidad, siendo el referente para el fin de la Edad Moderna y el principio de la Edad Contemporánea. Tuvo como origen el hartazgo del pueblo francés, que vivía una monarquía absoluta, y su fin fue establecer la primera República Francesa. De este movimiento surgió la Declaración de los Derechos del Hombre y del Ciudadano y en el momento en que la Asamblea Nacional Constituyente la adoptó, se dio el primer paso para la redacción de la Constitución de la República de Francia. La Declaración proclama que a todos los ciudadanos se les deben garantizar los ideales de libertad, igualdad y fraternidad. Los artículos más característicos y relevantes para el libre desarrollo de la personalidad son:

Artículo $1^{\circ}$

Los hombres han nacido, y continúan siendo, libres e iguales en cuanto a sus $\operatorname{derechos}(. .$.

Artículo $4^{\circ}$

(...) El ejercicio de los derechos naturales de cada hombre no tiene otros límites que los necesarios para garantizar a cualquier otro hombre el libre ejercicio de los mismos derechos; y estos límites sólo pueden ser determinados por la ley.

Por tanto, en el artículo $4^{\circ}$ es evidente la función negativa del Estado de no injerencia en las esferas personales del individuo y la función positiva garante de la pacífica conviven- 
cia social. Asimismo, establece la reserva de ley y los derechos de terceros como únicos límites a los derechos individuales.

Lo anterior es un indicio histórico del reconocimiento y aceptación de la personalidad humana y la autonomía en el desarrollo durante la vida del individuo. Es así que podemos observar que la transformación y evolución de la sociedad internacional ha reconocido el libre desarrollo de la personalidad, potenciándolo en el reconocimiento de la dignidad humana y su desarrollo social.

¿En qué consiste el derecho humano al libre desarrollo de la personalidad, según la Suprema Corte de Justicia de la Nación?

DERECHO AL LIBRE DESARROLLO DE LA PERSONALIDAD. BRINDA PROTECCIÓN A UN ÁREA RESIDUAL DE LIBERTAD QUE NO SE ENCUENTRA CUBIERTA POR LAS OTRAS LIBERTADES PÚBLICAS. (Tesis: 1a./J. 5/2019, (10ª), p. 487).

La Constitución Mexicana otorga una amplia protección a la autonomía de las personas, al garantizar el goce de ciertos bienes que son indispensables para la elección y materialización de los planes de vida que los individuos se proponen.

Así, en términos generales, puede decirse que los derechos fundamentales tienen la función de "atrincherar" esos bienes contra medidas estatales o actuaciones de terceras personas que puedan afectar la autonomía personal. De esta manera, los derechos incluidos en ese "coto vedado" están vinculados con la satisfacción de esos bienes básicos que son necesarios para la satisfacción de cualquier plan de vida. En este orden de ideas, el bien más genérico que se requiere para garantizar la autonomía de las personas es precisamente la libertad de realizar cualquier conducta que no perjudique a terceros. En este sentido, la Constitución y los tratados internacionales reconocen un catálogo de "derechos de libertad" que se tra- ducen en permisos para realizar determinadas acciones que se estiman valiosas para la autonomía de las personas (expresar opiniones, moverse sin impedimentos, asociarse, adoptar una religión u otro tipo de creencia, elegir una profesión o trabajo, etcétera), al tiempo que también comportan límites negativos dirigidos a los poderes públicos y a terceros, toda vez que imponen prohibiciones de intervenir u obstaculizar las acciones permitidas por el derecho fundamental en cuestión. Ahora bien, el derecho al libredesarrollo de la personalidad brinda protección a un "área residual de libertad" que no se encuentra cubierta por las otraslibertades públicas. En efecto, estos derechos fundamentales protegen la libertad de actuación humana de ciertos "espacios vitales" que, de acuerdo con la experiencia histórica, son más susceptibles de ser afectados por el poder público; sin embargo, cuando un determinado "espacio vital" es intervenido a través de una medida estatal y no se encuentra expresamente protegido por un derecho de libertad específico, las personas pueden invocar la protección del derecho al libre desarrollo de la personalidad. De esta manera, este derecho puede entrar en juego siempre que una acción no se encuentre tutelada por un derecho de libertad específico.

Los hechos de la sentencia aludida -solo para efectos de contextualización, porque no constituyen el motivo de este trabajo- consistieron en que la parte quejosa, Sociedad Mexicana de Autoconsumo Responsable y Tolerante (SMART), solicitó a la Comisión Federal para la Protección de Riesgos Sanitarios (COFEPRIS) la autorización para la importación y adquisición de semilla de marihuana para su final autoconsumo para fines lúdicos y recreativos, así como para ejercer los derechos correlativos al autoconsumo, tales como la siembra, cultivo, cosecha, preparación, acondicionamiento, posesión, transporte, empleo y en general todo uso relacionado con el consumo lúdico y personal (exclyendo actos de comercio).

Ante la negativa, promovió amparo indirecto reclamando los artículos 235, 237, 245, 
fracción I, 247, último párrafo, y 248 de la Ley General de Salud, sin que se solicitara la adquisición de la planta o sus productos, lo que mostraba la intención de realizar por sí mismo la siembra, cultivo, cosecha, preparación, acondicionamiento y consumo, limitando la función de terceros a la entrega de la semilla.

El sistema de prohibición menoscaba de manera importante el derecho al libre desarrollo de la personalidad, al no encontrarse que el consumo de marihuana genere consecuencias que por su gravedad afecten de manera importante a la salud o al orden público (Silva, K., 2014).

En la sentencia, que al sumar cinco amparos de sendos quejosos en el mismo sentido sentó jurisprudencia, se destaca que la Constitución Federal protege ampliamente la autonomía de las personas al garantizar el goce de ciertos bienes que son indispensables para la elección y materialización de sus planes de vida, y el bien más elemental es la libertad de llevar a la práctica cualquier conducta en tanto que no perjudique a terceros. (Díez- Picazo, 2003, p. 6466).

La Constitución Federal y los Tratados Internacionales reconocen un catálogo de derechos de libertad que son valiosos para la autonomía personal, como la libertad de expresión, de tránsito, de asociación, de reunión, de adoptar relaciones o creencias, de elegir la profesión o el trabajo, entre otras, y que simultáneamente sirven como límites a la autoridad.

Un razonamiento muy interesante dentro de esta jurisprudencia es el que se hace en el sentido de que el libre desarrollo de la personalidad salvaguarda un área residual que no cubren las restantes libertades públicas, que protegen espacios vitales de la libertad humana que -citando el caso Elfes en el que el Tribunal Constitucional Alemán consideró que la negativasinjustificación alguna por parte de las autoridades públicas de negar la expedición del pasaporte a un ciudadano alemán (Wilhelm
Elfes) iba en contra de la libertad general consagrada en la Constitución Alemana, de donde se desprende la libertad para viajar, y que reserva una esfera para la planeación de la vida privada del individuo, que constituye un ámbito inviolable de libertad humana son más susceptibles de ser afectados por el poder público, pero cuando no se encuentra protegido un espacio vital con esas libertades, entra en juego aquél otro (libre desarrollo de la personalidad).

En relación a la jurisprudencia comparada, la Suprema Corte de Justicia de la Nación señala que este derecho comporta "un rechazo radical de la siempre presente tentación del paternalismo del Estado, que cree saber mejor que las personas lo que conviene a éstas y lo que deben hacer con sus vidas". Supone "la proclamación constitucional de que, siempre que se respeten derechos de los demás, cada ser humano es el mejor juez de sus propios intereses" (Caso Elfes).

Siguiendo con la observación de la jurisprudencia, se reconoce que el derecho fundamental al libre desarrollo de la personalidad deriva de la dignidad humana reconocida en el artículo primero de la Constitución Política de los Estados Unidos Mexicanos:

Artículo 1o. En los Estados Unidos Mexicanos todas las personas gozarán de los derechos humanos reconocidos en esta Constitución y en los tratados internacionales de los que el Estado Mexicano sea parte, así como de las garantías para su protección, cuyo ejercicio no podrá restringirse ni suspenderse, salvo en los casos y bajo las condiciones que esta Constitución establece.

Las normas relativas a los derechos humanos se interpretarán de conformidad con esta Constitución y con los tratados internacionales de la materia, favoreciendo en todo tiempo a las personas la protección más amplia.

Todas las autoridades, en el ámbito de sus competencias, tienen la obligación de pro- 
mover, respetar, proteger y garantizar los derechos humanos de conformidad con los principios de universalidad, interdependencia, indivisibilidad y progresividad. En consecuencia, el Estado deberá prevenir, investigar, sancionar y reparar las violaciones a los derechos humanos en los términos que establezca la ley.

Está prohibida la esclavitud en los Estados Unidos Mexicanos. Los esclavos del extran jero que entren al territorio nacional alcanzarán, por este solo hecho, su libertad y la protección de las leyes. Queda prohibida toda discriminación motivada por origen étnico o nacional, el género, la edad, las discapacidades, la condición social, las condiciones de salud, la religión, las opiniones, las preferencias sexuales, el estado civil o cualquier otra que atente contra la dignidad humana y tenga por objeto anular o menos cabar los derechos y libertades de las personas.

Con base en lo anterior, la Suprema Corte de Justicia de la Nación en su interpretación señala que todas las personas son iguales ante la ley, sin que pueda prevalecer discriminación alguna por razones étnicas o de nacionalidad, raza, sexo, religión o cualquier otra condición o circunstancia personal o social que atente contra la dignidad humana y que, junto con los instrumentos internacionales en materia de derechos humanos suscritos por México, reconocen el valor superior de la dignidad humana, es decir, que en el ser humano hay una dignidad que debe ser respetada en todo caso, constituyéndose como un derecho absolutamente fundamental, base y condición de todos los demás:

(...) el derecho a ser reconocido y a vivir en y con la dignidad de la persona humana, y del cual se desprenden todos los demás derechos, en cuanto son necesarios para que los individuos desarrollen integralmente su personalidad, dentro de los que se encuentran, entre otros, el derecho a la vida, a la integridad física y psíquica, al honor, a la privacidad, al nombre, a la propia imagen, al libre desarrollo de la personalidad, al estado civil y el propio derecho a la dignidad personal. Además, aun cuando estos derechos personalísimos no se enuncian expresamenteen la Constitución Generalde la República, están implícitos en los tratados internacionales suscritos por México y, en todo caso, deben entenderse como derechos derivados del reconocimiento al derecho a la dignidad humana, pues sólo a través de su pleno respeto podrá hablarse de un ser humano en toda su dignidad (DIGNIDAD HUMANA. EL ORDEN JURÍDICO MEXICANO LA RECONOCE COMO CONDICIÓN Y BASE DE LOS DEMÁS DERECHOS FUNDAMENTALES. Tesis. P. LXV/2009, (9a), p.8).

Definición que se encuentra de cierta forma sintetizada:

(...) el reconocimiento del Estado sobre la facultad natural de toda persona a ser individualmente como quiere ser, sin coacción ni controles injustificados, con el fin de cumplir las metas u objetivos que se ha fijado, de acuerdo a sus valores, ideas, expectativas, gustos, etcétera (Carbonell, 2018).

\section{Conclusión}

Para tener una idea clara y sencilla del concepto genérico de libertad, se estima que pueden ser de gran utilidad un par de ejemplos. El filósofo danés Søren Kierkegaard, que combinó fe y filosofía, sostuvo que como el ser humano no está programado por la naturaleza para cumplir determinadas funciones biológicas o zoológicas, puede (o tiene) que elegir entre una amplia gama de opciones, lo que significa libertad, pero que a la vez genera incertidumbre, y eso nos hace buscar algo que nos dé tranquilidad (fe) (Savater, 2008). Por su parte, el filós fo alemán Arthur Schopenhauer sostuvo que la libertad es un concepto negativo que representa la falta de todo impedimento y obstáculo, que puede responder a una libertad física, intelectual y moral (Schopenhauer, A., 2001, p. 7). 
¿El libre desarrollo de la personalidad es un derecho emergente?

El resultado de la observación de la jurisprudencia señalada permite entender que sí constituye un derecho emergente, pues los derechos humanos emergentes pretenden traducir nuevas necesidades de la humanidad en nuevos derechos y por otra parte son derechos que emergen tras haber permanecido "sumergidos" en el olvido o en la indiferencia de los estados y del conjunto del sistema internacional.

El libre desarrollo de la personalidad encuadra perfectamente como derecho emergente, ya que ha surgido mediante la interpretación constitucional de la Suprema Corte de Justicia de la Nación, motivada por demandas de la sociedad. Es explicable que haya estado sumergido en la opacidad al tomar en cuenta que las necesidades sociales y formas de pensar de inicios del siglo XX distan de las actuales.

Se estima también que no es posible precisar sus alcances de una vez y para siempre, porque su esencia radica en el individualismo, en los juicios subjetivos de valor que cada persona tenga sobre sí misma, su plan de vida y todo lo que ello en conjunto implica, es decir, lo que para uno es importante para su proyecto vital, puede ser irrelevante o muy distinto para los demás. Incluso, es un concepto dinámico que irá transformándose a la velocidad que evolucionen las necesidades de la sociedad, por

\section{Referencias}

Carbonell, M. (2008). La libertad. Dilemas, retos y tensiones. México: UNAM, CNDH.

Schopenhauer, A. (2001). La libertad. México: Ediciones Coyoacán.

Savater, F. (2008), La aventura de pensar. Barcelona: De bolsillo.

Tesis: 1a./J. 5/2019. Semanario Judicial de la Federación y su Gaceta, Décima Época, t. I, Febrero 2019, p. 487. Con registro de identificación 2019355.

Tesis: P. LXV/2009. Semanario Judicial de la Federación y su Gaceta, Novena Época, t. XXX, Diciembre 2009, p. 8. Con registro de identificación 165813.

Sentencia de la Primera Sala de fecha 16 de enero de $195 \%$. Tribunal Constitucional Federal Alemán, 1BvR 253/56. lo que fijar sus alcances en un concepto estático sería tanto como limitar dicho derecho.

Lo anterior podría llevarnos a nuevas interrogantes: Si hoy el libre desarrollo de la personalidad protege el autoconsumo de marihuana, el divorcio sin causa, el divorcio sin esperar un año, la identidad personal, sexual y de género, qué bienes jurídicos protegerá mañana ¿poligamia? Hace décadas ese derecho estaba limitado, en ese entonces no se contemplaban los alcances que ahora tiene, lo que nos conduce a preguntarnos qué bienes o derechos tutelará más adelanteque ahora tal vez parezcan inalcanzables. No obstante, es precisamente eso, la confianza en que se realizará algo en el futuro, lo que nos permite seguir avanzando en la construcción del sistema jurídico mexicano.

Las ideas anteriores nos invitan a reflexionar en los retos y expectativas del libre desarrollo de la personalidad en nuestra sociedad, lo que podrá ser materia de otro trabajo. El libre desarrollo de la personalidad es un derecho que garantiza la libertad general de acción humana sin impedimentos de ninguna naturaleza, que nos permite optar, decidir y llevar a cabo nuestro plan de vida de acuerdo a nuestros gustos, intereses, ideas, estilos, valores, cultura, etcétera, restringido por las limitaciones del orden jurídico y los derechos de los demás.

Silva, K. (2014). Zaldívar y su proyecto sobre uso recreativo de la marihuana. [En línea]. Consultado el 30 de noviembre de 2019. Disponible en: https://eljuegodela corte.nexos.com.mx/?p=5043

Carbonell, M. (2018). Dignidad Humana. [En línea]. Consultado el 15 de diciembre de 2019. Disponible en: https://revistas.juridicas.unam.mx/index.php/hechosderechos/article/view/12985/14530

Hernández, A. (2018). Derecho al libre desarrollo de la personalidad. [En línea]. Consultado el 30 de Noviembre de 2019. Disponible en: https://www.jornada.com.mx/ 2018/01/19/politica/017a2pol

Díez-Picazo, M. (2003). Sistema de Derechos Fundamentales, serie "Derechos Fundamentales y Libertades 
Públicas. [En línea]. Consultado el 15 de Diciembre de 2019. Disponible en: https://www.cortesaragon.es/fileadmin/_ DMZMedia/biblioteca/boletinNovedades/201406/5\%. pdf

Constitución Política de los Estados Unidos Mexicanos (1917). Consultado el 10 de diciembre de 2019. Disponible en: https:/www.scjn.gob.mx/sites/default/files/pagina/documentos/2020-05/CPEUM_08052020.pdf

Ley General de Salud (2006). [En línea]. Consultado el 10 de diciembre de 2019. Disponible en: http:// www.diputados.gob.mx/LeyesBiblio/ref/lgs.htm. Declaración Universal de Derechos Humanos de 1948. [En línea]. Consultado el 20 de enero de 2020. Disponible en:
https://ohchr.org/EN/UDHR/Documents/UDHR-Translations/spn.pdf

Declaración Universal de Derechos Humanos Emergentes, (2007). [En línea]. Consultado el 10 de diciembre de 2019. Disponible en: https://catedraunescodhunam. $\mathrm{mx} / \mathrm{catedra} / \mathrm{CONACYT/04}$ _Docentes_UdeO_ubicar_el_de_alumnos/Contenidos/Lecturas\%20obligatorias/M.5_cont_3_DUDHE.pdf.

Declaración de los Derechos del Hombre y del Ciudadano de 1789. [En línea]. Consultado el 10 de diciembre de 2019. Disponible en: http://www.pudh.unam. $\mathrm{mx} /$ declaracion_DH_hombre_ciudadano.html 